\title{
Infrared multiple photon dynamics and spectroscopy of cationic PABA and its dehydroxylated fragment ion
}

\author{
Jos Oomens, ${ }^{a}$ David T. Moore, ${ }^{a}$ Gerard Meijer $^{a b}$ and Gert von Helden ${ }^{b}$ \\ a FOM Institute for Plasma Physics “Rijnhuizen”, Edisonbaan 14, 3439MN, Nieuwegein, \\ The Netherlands.E-mail: joso@rijnh.nl \\ ${ }^{b}$ Fritz-Haber-Institut der Max-Planck-Gesellschaft, Faradayweg 4-6, D-14195 Berlin, Germany
}

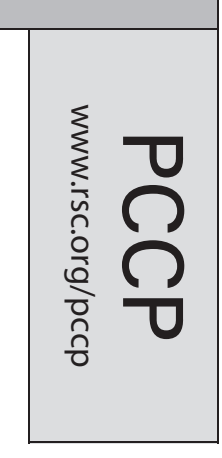

Received 24th November 2003, Accepted 18th December 2003

First published as an Advance Article on the web 22nd January 2004

\begin{abstract}
The gas-phase infrared spectra of cationic para-amino benzoic acid (PABA, $\left.\mathrm{H}_{2} \mathrm{~N}-\mathrm{C}_{6} \mathrm{H}_{4}-\mathrm{COOH}\right)$ and its dehydroxylated fragment ion, the para-amino benzoyl (PABz, $\mathrm{H}_{2} \mathrm{~N}-\mathrm{C}_{6} \mathrm{H}_{4}-\mathrm{CO}$ ) cation, are presented in the $500-2500 \mathrm{~cm}^{-1}$ range. The spectra are obtained via mass-selective infrared multiple photon dissociation (IRMPD) using a continuously tunable free electron laser. Although both spectra are in good overall agreement with results from density functional theory, some interesting discrepancies are also observed. Particularly in the spectrum of the PABz cation, the strongly anharmonic $\mathrm{NH}_{2}$ out-of-plane bending mode causes substantial deviations from theory. The relative intensity of this out-of-plane bending mode in both the PABA and PABz cation spectra is much lower than calculated, similar to what has been reported for cationic aniline. For the $\mathrm{PABz}$ cation, two distinct fragmentation products are observed and recording the infrared spectra on these individual decay channels yields information on the dissociation mechanism. Moreover, it is experimentally shown that the decay rate into the two competing channels can qualitatively be described using statistical models.
\end{abstract}

\section{Introduction}

Infrared spectra of gas-phase molecular ions are notoriously difficult to measure as space charge limits the ion densities that can be obtained. ${ }^{1}$ A variety of small molecular ions has been studied in fast ion beams, both using direct absorption as well as indirect detection methods. In addition, small molecular ions can be produced rather efficiently in discharge sources, allowing for direct absorption methods to be applied, but larger molecular ions usually suffer from fragmentation in these sources, requiring the use of mass-selective techniques. However, the use of such methods generally reduces the obtainable densities such that inventive "action spectroscopy" schemes need to be applied.

On the other hand, the use of mass-selective methods has the advantage that it facilitates spectroscopic investigation of not only the parent ions but also of their derivatives, such as fragmentation products, which are otherwise difficult to study. Although fragmentation patterns are in general well documented in terms of mass distributions (see e.g. ref. 2 for polyaromatic species), i.e. in terms of the atomic compositions, the molecular structure of the fragments remains largely unknown. Nonetheless, the structure of these transient molecular fragments may be of wide interest in a variety of scientific fields, e.g. they may play a role as reaction intermediates or they may occur in low-density environments such as interstellar clouds. Obviously, obtaining infrared spectral information on such (ionic) molecular fragments would be a big step forward toward their structural characterization.

Infrared resonance enhanced multiple photon dissociation (IRMPD) spectroscopy has proven to be a useful variant of action spectroscopy (see e.g. ${ }^{3-8}$ ). Rather than measuring the direct absorption of infrared photons, detection is instead based on changes in the chemical composition of the sample, which, for example, can be diagnosed from its mass spectrum. Commonly, the dissociation yield, i.e. the fragment intensity over the total intensity, serves as the signal for infrared absorption. In combination with ion storage devices, such as simple quadrupole ion traps, ${ }^{9-11}$ this technique can be used to obtain infrared spectra of ionic species. Obviously, for the fragmentation of most molecular species, intense laser sources are required since the absorption of many photons is necessary to induce dissociation.

In the 1990's, this method has been applied using $\mathrm{CO}_{2}$ lasers, ${ }^{12-14}$ which however, have only limited and discontinuous wavelength tunability. In addition, the technique has been applied to non-covalently bound ionic species ${ }^{15,16}$ using infrared sources with lower output power. Over the past few years, we have employed a free electron laser (FEL) to study the infrared spectroscopy of large gas phase molecular ions. ${ }^{17}$ The ions are mass-selected and stored in a quadrupole ion trap, where they are subjected to the intense pulsed output of an FEL. More recently, it was shown that the FEL-based approach can also be applied using more sophisticated ion trapping devices, in particular linear multipole traps ${ }^{18,19}$ and Fourier transform ion cyclotron resonance (FTICR) mass spectrometers. $^{20,21}$

As an example of fragment ion infrared spectroscopy, we recently recorded the first infrared spectrum of the benzoyl cation, ${ }^{22}$ which has been known in its neutral radical form since the 19th century, ${ }^{23}$ but for which no spectroscopic data had been reported. In the current work, we present a more extensive study of the infrared spectra of the para-amino benzoic acid (PABA) cation and its dehydroxylated fragment, the para-amino benzoyl (PABz) cation.

Combining amino and carboxylic acid functional groups, PABA is an interesting molecule forming a hybrid of two benchmark molecules, benzoic acid and aniline. The structures of PABA and PABz are given in Fig. 1. Both systems undergo substantial structural changes upon ionization as is evident from DFT calculations performed at the B3LYP level of theory (vide infra). PABA has been widely used in sunscreens and is also known under exotic names as "Sunbrella" and "Super Shade". Its jet-cooled UV spectrum was recorded over 

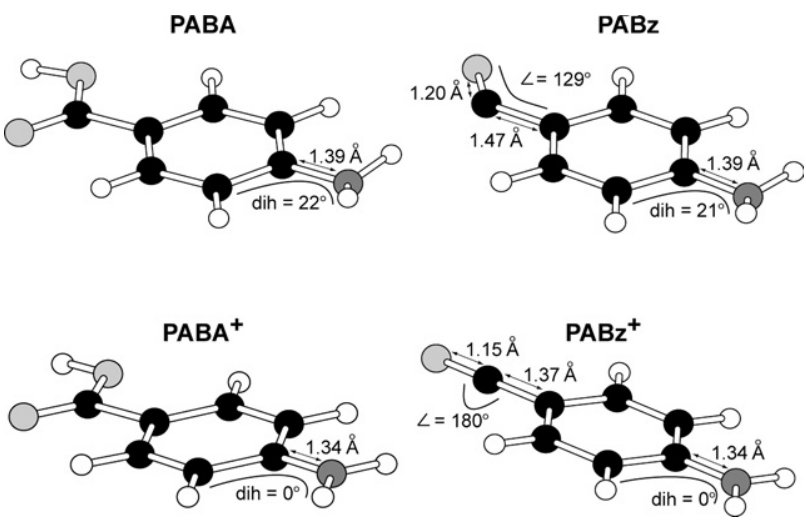

Fig. 1 Structures of neutral and ionic PABA and PABz. Main structural changes upon ionization, derived from DFT calculations at the B3LYP/D95(d,p) level, are indicated (dih = dihedral angle). The systems become planar and the carbonyl side chain in PABz becomes linear. In addition, due to conjugation several side chain bond lengths shorten.

a decade ago. ${ }^{24}$ Recently, the gas-phase infrared spectra of cationic aniline ${ }^{25}$ and benzoic acid, ${ }^{26}$ as well as that of neutral PABA, ${ }^{27}$ were investigated with the use of an FEL. To our knowledge, no infrared spectra have to date been reported for the PABA cation nor for the PABz cation.

In the present study, the dissociation dynamics of the $\mathrm{PABz}$ cation were found to be especially interesting as there are two competing dissociative decay channels with different barriers within the range accessible through infrared multiple photon excitation. By studying the anharmonic redshifting with a continuously wavelength tunable FEL, one obtains a clear experimental view of the statistical dissociation dynamics involved. In addition, this example provides detailed experimental insight into the IRMPD technique, in particular into the effects of anharmonicity and dissociation threshold on observed band positions.

\section{Experimental}

The experimental apparatus is described in detail elsewhere ${ }^{17}$ and only a brief outline will be given here. It consists of a Paul-type quadrupole ion trap ${ }^{9-11}$ combined with a timeof-flight (TOF) mass spectrometer ${ }^{28}$ and the experiments are carried out at the Free Electron Laser for Infrared eXperiments (FELIX) facility. ${ }^{29}$ Using the mass-selective instability properties of the trap, one can eject species below a cut-off mass-to-charge ratio, which is determined by the rf amplitude. Hence, the heaviest mass ion in the trap can easily be isolated. The isolated parent ion is then irradiated with an FEL infrared pulse. The contents of the trap are subsequently pulseextracted into the TOF mass spectrometer and fragment ions are observed whenever the infrared laser has induced resonance enhanced multiple photon dissociation.

Under typical operation conditions, FELIX delivers $5 \mu \mathrm{s}$ long macropulses with an energy of $100 \mathrm{~mJ}$ at a $5 \mathrm{~Hz}$ repetition rate. ${ }^{29}$ The wavelength is continuously tunable over the entire far to mid-infrared range $\left(40-2000 \mathrm{~cm}^{-1}\right)$. In addition, the wavelength range between $2000-3500 \mathrm{~cm}^{-1}$ is accessible by operating FELIX on the third harmonic, which is accomplished by replacing one of the gold-coated cavity mirrors with a dielectrically coated mirror, reflective at the third harmonic and transparent to the fundamental wavelength. Each macropulse consists of a $1 \mathrm{GHz}$ train of micropulses and the laser bandwidth, typically $0.5 \%$ of the central wavelength, is determined by the micropulse width. The wavelength is calibrated with a grating spectrometer and is estimated to be accurate to within $0.2 \%$ and hence, we believe the accuracy of the reported band centers to be about $0.5 \%$. Note also that the IRMPD process induces a vibrational-mode-dependent redshift, which was found to be typically $1.5 \%$ for a variety of polyaromatic ions. ${ }^{30}$

Solid PABA is vaporized in an oven at about $70^{\circ} \mathrm{C}$ and ionized in the center of the ion trap using the focused output of an ArF excimer laser (Neweks PSX-100). The focused UV beam $\left(4 \mathrm{~mJ}\right.$ pulse $\left.\mathrm{e}^{-1}\right)$ causes extensive fragmentation of PABA and no parent ion can be detected. Thus, the $m / z=120 \mathrm{u}$ $e^{-1}$ fragment ion (dubbed m120 hereafter) is the heaviest species in the trap. This fragment is formed by removal of the hydroxyl group from PABA, resulting in the PABz closed-shell fragment ion. Such ionization/fragmentation dynamics are similar to what has been observed for benzoic acid ${ }^{22}$ and can be understood from ion energetics

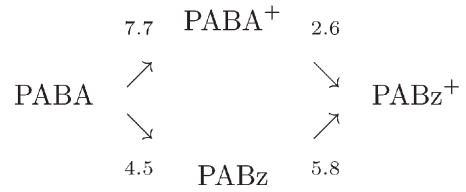

where all values represent DFT calculated adiabatic dissociation and ionization energies in eV. Although experimental values are available for some of the energies in the scheme, ${ }^{24,31}$ we use only calculated values to obtain a consistent picture. Using the ArF excimer laser with a photon energy of $6.4 \mathrm{eV}$, the decay channel via neutral PABz is preferred since it can proceed via two one-photon processes. Under our experimental conditions, no direct UV induced $\mathrm{PABA}^{+}$is observed since it can only be formed via direct two-photon absorption. Hence, the PABz ion is the heaviest species in the trap and it can be isolated using a simple rf amplitude sequence, after which its IRMPD spectrum can be recorded.

In order to generate the intact PABA ion, we make use of ion-molecule reactions occurring in the trap, which induce chemical ionization of PABA. If one allows for a delay on the order of $100 \mathrm{~ms}$ after the UV pulse, neutral PABA vapor will undergo charge transfer with small $\dagger$ UV laser produced ionic fragments in the trap. Hence, the PABA cation at m137 becomes the heaviest species in the trap and can be isolated by pulsing the rf voltage to a slightly higher amplitude after the charge transfer delay. This strategy has previously been used in experiments on benzoic acid, ${ }^{26}$ where it has been described in more detail.

PABA was purchased from Aldrich and used without further purification. Deuterated $\mathrm{PABz}$ was generated from D-substituted PABA, which is obtained by $\mathrm{H}-\mathrm{D}$ exchange in a solution of PABA in $\mathrm{D}_{2} \mathrm{O}$. A small amount $(\approx 5 \%)$ of methanol was added to increase the solubility of PABA and the solution was heated to about $60^{\circ} \mathrm{C}$. After a few hours, dehydration of the solution yielded a sample of PABA with a partly D-substituted amino-group.

\section{Results and discussion}

Upon resonant infrared irradiation of $\mathrm{PABA}^{+}$, two main fragmentation products are observed at $\mathrm{m} 120$ and $\mathrm{m} 92$, corresponding to loss of the $\mathrm{OH}$ group (forming $\mathrm{PABz}^{+}$) and loss of the entire carboxylic acid side chain, respectively. $\mathrm{PABz}^{+}$ is found to fragment into channels $\mathrm{m} 92$ and $\mathrm{m} 65$; the $\mathrm{m} 92$ fragment is the same as that found from $\mathrm{PABA}^{+}$and the m65 fragment probably corresponds to $\mathrm{C}_{5} \mathrm{H}_{5}{ }^{+}$. For discussion of the infrared spectra, the signal in all fragment channels is summed and the total fragment yield serves as the signal in the infrared action spectra. Later, in Section 3.4, we shall

$\dagger$ Charge transfer from $\mathrm{PABz}^{+}$is unlikely because of the ratio of ionization potentials of $\mathrm{PABA}$ and $\mathrm{PABz}$ as apparent from the reaction scheme. 
discuss the dissociation dynamics in more detail and analyze the individual fragment channels.

\subsection{Infrared spectrum of the PABA cation}

Upon resonant infrared irradiation, the PABA cation dissociates mainly into the m120 mass channel, i.e. the PABz cation. Under our experimental conditions, fragmentation into the m92 channel was typically less than $20 \%$ of that into $\mathrm{m} 120$. The intensities in the two channels were added to give the IRMPD spectrum of the intact PABA cation as displayed in Fig. 2.

The experimental spectrum is compared with a DFT calculation using the B3LYP functional and D95(d,p) basis set. ${ }^{32}$ Frequencies were scaled by 0.98 , as was found appropriate for the B3LYP functional in combination with a basis set including polarization functions. ${ }^{33}$ As in cationic aniline, ${ }^{25,34-38}$ removal of one of the nitrogen lone-pair electrons and conjugation of the remaining unpaired electron to the aromatic $\pi$-system, leads to $\mathrm{sp}^{2}$ hybridization on the $\mathrm{N}$ atom and hence theory predicts a planar structure (see Fig. 1). In general, agreement between experiment and theory is found within $1.5 \%$ and the assignment of most bands as listed in Table 1 is straightforward, confirming the planar structure of the ion. One should keep in mind that the multiple photon excitation process can cause a slight red shift of the bands. Unlike the PABz cation (see below), no bands are observed that have no calculated counterpart. Moreover, even subtle details of the spectrum are well reproduced by the calculation, such as the strong feature around 1350 $\mathrm{cm}^{-1}$, which appears to consist of more than one band, or the weak feature around $1000 \mathrm{~cm}^{-1}$. All mid-infrared bands

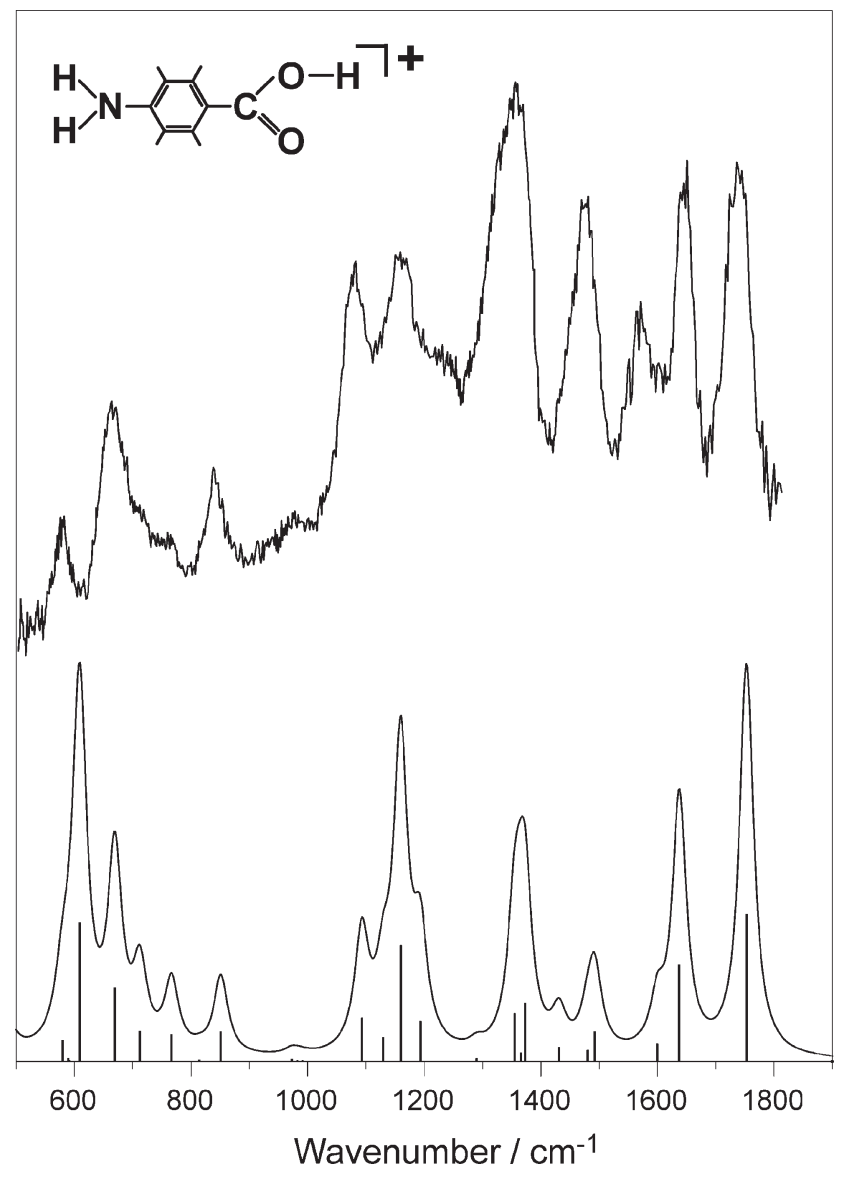

Fig. 2 IRMPD spectrum of the intact PABA cation compared with the B3LYP/D95(d,p) calculated spectrum.. of the PABA cation, including the $\mathrm{CO}$ stretching band, fall in the fundamental FELIX wavelength range.

Some discrepancies in relative intensities are observed, most notably on the $\mathrm{NH}_{2}$ out-of-plane bending mode, i.e. the "inversion mode' of the amino group $\ddagger$, near $600 \mathrm{~cm}^{-1}$. From the last column in Table 1, it is noted that the deviation between calculated and observed frequency is relatively large for this band $(5.1 \%)$. In terms of the relative intensity of this band, a behaviour similar to that of cationic aniline $e^{25}$ is observed. The discrepancy in the intensity of the inversion mode is likely caused by the strong anharmonicity of this mode. Interestingly, it may be noted that either the harmonic DFT calculation has problems predicting the correct intensity and frequency of such modes or that the experiment has problems with this mode as the large anharmonicities make the multiple photon excitation process relatively inefficient. Although from the present multiple photon experiment it is difficult to judge which of these effects causes the deviation, the linear absorption spectrum of cationic aniline showed that DFT overestimates the intensity of the $\mathrm{NH}_{2}$ inversion mode by a factor of about four. ${ }^{25}$

\subsection{Infrared spectrum of the para-amino benzoyl cation}

The spectrum of the $\mathrm{PABz}$ cation as shown in Fig. 3 is obtained by summing the fragment yields in the $\mathrm{m} 92$ and m65 mass channels. Band centers are listed in Table 2 along with the results of a DFT calculation using the B3LYP functional and the D95(d,p) basis set (frequencies scaled by 0.98 ). All the main calculated bands are readily identified in the experimental spectrum. The DFT calculation predicts a planar geometry with a $180^{\circ} \mathrm{CCO}$ angle to be the lowest energy structure for the PABz cation (see Fig. 1). This is similar on the one hand to the benzoyl cation, where removal of the unpaired electron from the carboxylic $\mathrm{C}$ atom changes its hybridization to sp yielding a linear $\mathrm{CCO}$ unit $^{22}$ and, on the other hand, to ionic aniline, where removal of one of the lone-pair electrons on the $\mathrm{N}$ atom leads to $\mathrm{sp}^{2}$ hybridization and hence a planar structure. ${ }^{25}$ The reasonably good overall agreement with the experimental spectrum, as shown in Fig. 3, indicates that this geometry with $C_{2 \mathrm{v}}$ symmetry is indeed correct. Four interesting regions in the spectrum are discussed further below.

The $400-600 \mathrm{~cm}^{-1}$ region. In the long wavelength region, the FELIX beam diameter increases substantially, which leads to a reduced transmission through the holes drilled in the ion trap. Although we attempted to correct the line intensities in the spectrum for the reduced infrared power, deviations are possible, especially since the linear power correction is not necessarily correct. Nonetheless, we suspect that the intensity of the $476 \mathrm{~cm}^{-1}$ band is correctly reproduced and that the deviation from theory is caused by the strongly anharmonic nature of the involved vibration, the out-of-plane $\mathrm{NH}_{2}$ bending mode, corresponding to the inversion mode in the non-planar neutral species. Below, we will further elaborate on this assumption.

The $900-1200 \mathrm{~cm}^{-1}$ region. In this spectral region, there is clearly less intensity calculated than observed. In fact, only the band observed at $1176 \mathrm{~cm}^{-1}$ can be assigned as the in-plane $\mathrm{CH}$ bending mode calculated at $1180 \mathrm{~cm}^{-1}$. Although the bands observed to the red are severely congested (which makes their band positions in Table 2 somewhat speculative), it can be clearly seen that they cannot be explained by the DFT calculation. Several explanations for the additional structure are

\pm Strictly speaking, since the cation is planar and the vibrational potential is barrierless, the term "inversion" mode is incorrect. However, in the similar case of aniline, it is often referred to as such for reasons of correspondence with the non-planar neutral ground state. We shall use this terminology for PABz and PABA as well. 
Table 1 Infrared spectrum of the para-amino benzoic acid cation

\begin{tabular}{|c|c|c|c|c|c|c|}
\hline \multirow[b]{2}{*}{ Mode $^{b}$} & \multirow[b]{2}{*}{ Symmetry } & \multicolumn{2}{|c|}{$\mathrm{B} 3 \mathrm{LYP} / \mathrm{D} 95(\mathrm{~d}, \mathrm{p})^{a}\left(C_{\mathrm{s}}\right)$} & \multicolumn{2}{|c|}{ Observed } & \multirow{2}{*}{$\begin{array}{l}\mathrm{O}-\mathrm{C} \\
\%\end{array}$} \\
\hline & & $\nu$ & $\operatorname{Int}^{c}$ & $\nu$ & $\operatorname{Int}^{c}$ & \\
\hline$\delta($ ring $) \alpha(\mathrm{OCO})$ & $\mathrm{a}^{\prime}$ & 579.5 & 30 & & & \\
\hline$\beta^{\prime}\left(\mathrm{NH}_{2}\right)$ & $a^{\prime \prime}$ & 608.9 & 198 & 578 & 0.22 & -5.1 \\
\hline$\beta^{\prime}(\mathrm{CH}) \beta^{\prime}(\mathrm{OH})$ & $a^{\prime}$ & 669.4 & 105 & 667 & 0.42 & -0.3 \\
\hline$\delta($ ring $) \alpha(\mathrm{OCO})$ & $\mathrm{a}^{\prime}$ & 712.5 & 43 & 710 & 0.11 & -0.4 \\
\hline$\beta^{\prime}(\mathrm{CH}) \beta^{\prime}(\mathrm{CC})$ & $a^{\prime \prime}$ & 766.7 & 38 & 766 & 0.11 & -0.1 \\
\hline$\beta^{\prime}(\mathrm{CH})$ & $a^{\prime \prime}$ & 851.2 & 42 & 845 & 0.29 & -0.7 \\
\hline$\beta(\mathrm{CH}) \delta($ ring $)$ & $a^{\prime}$ & 972.6 & 3 & $\approx 977$ & 0.15 & $\approx 0.4$ \\
\hline$\beta(\mathrm{CH}) \sigma(\mathrm{C}-\mathrm{O})$ & $\mathrm{a}^{\prime}$ & 1093.0 & 62 & 1081 & 0.67 & -1.1 \\
\hline$\beta(\mathrm{CH})$ & $\mathrm{a}^{\prime}$ & 1129.5 & 34 & & & \\
\hline$\beta(\mathrm{CH}) \beta(\mathrm{OH})$ & $\mathrm{a}^{\prime}$ & 1159.5 & 165 & 1161 & 0.67 & 0.1 \\
\hline$\beta(\mathrm{CH}) \beta(\mathrm{OH})$ & $\mathrm{a}^{\prime}$ & 1193.3 & 57 & $\approx 1230$ & & $\approx 3.1$ \\
\hline$\beta(\mathrm{CH}) \beta(\mathrm{OH}) \beta(\mathrm{NH})$ & $\mathrm{a}^{\prime}$ & 1355.5 & 68 & 1328 & 0.67 & -2.1 \\
\hline$\beta(\mathrm{CH}) \beta(\mathrm{NH}) \sigma(\mathrm{CC})$ & $\mathrm{a}^{\prime}$ & 1365.7 & 12 & & & \\
\hline$\beta(\mathrm{OH}) \beta(\mathrm{NH}) \sigma(\mathrm{CC})$ & $\mathrm{a}^{\prime}$ & 1372.7 & 83 & 1363 & 0.80 & -0.7 \\
\hline$\beta(\mathrm{CH}) \sigma(\mathrm{CC}) \beta\left(\mathrm{NH}_{2}\right)$ & $\mathrm{a}^{\prime}$ & 1431.3 & 20 & & & \\
\hline$\beta(\mathrm{CH}) \sigma(\mathrm{CC})$ & $\mathrm{a}^{\prime}$ & 1480.4 & 16 & & & \\
\hline$\beta(\mathrm{CH}) \alpha\left(\mathrm{NH}_{2}\right) \sigma(\mathrm{CN})$ & $\mathrm{a}^{\prime}$ & 1492.6 & 42 & 1476 & 0.82 & -1.1 \\
\hline$\beta(\mathrm{CH}) \sigma(\mathrm{CC}) \alpha\left(\mathrm{NH}_{2}\right)$ & $\mathrm{a}^{\prime}$ & 1599.9 & 25 & 1572 & 0.50 & -1.8 \\
\hline$\alpha\left(\mathrm{NH}_{2}\right)$ & $\mathrm{a}^{\prime}$ & 1637.8 & 138 & 1647 & 0.84 & -0.6 \\
\hline$\sigma(\mathrm{C}=\mathrm{O})$ & $\mathrm{a}^{\prime}$ & 1752.9 & 210 & 1740 & 1.00 & -0.7 \\
\hline
\end{tabular}

${ }^{a}$ DFT frequencies scaled by $0.98 .{ }^{b} \alpha=$ scissor; $\beta^{\prime}=$ out-of-plane bend; $\beta=$ in-plane bend; $\delta=$ deformation; $\sigma=$ stretch. ${ }^{c}$ Calculated intensity in $\mathrm{km} \mathrm{mol}^{-1}$. Observed intensity: relative

conceivable: (1) at a laser wavelength around $1080 \mathrm{~cm}^{-1}$, the very strong $\mathrm{CO}$ stretching mode can be reached via 2-photon absorption, which aids the multiphoton excitation process, as was already observed in the spectrum of the benzoyl cation. ${ }^{22}$ (2) The overtones of the out-of-plane bending modes are expected to lie in this region. Moreover, the overtone of the strongly anharmonic $\mathrm{NH}_{2}$ "inversion" mode falls in this region. Infrared spectra of $\mathrm{PABz}^{+}$with a partly $\mathrm{D}$-substituted amino group suggest that the additional spectral structure is indeed correlated with this mode (see below). Note that in the PABA ${ }^{+}$ spectrum, the corresponding frequency range, i.e. around 1200 $\mathrm{cm}^{-1}$, is strongly congested, which may obscure the presence of additional structures.

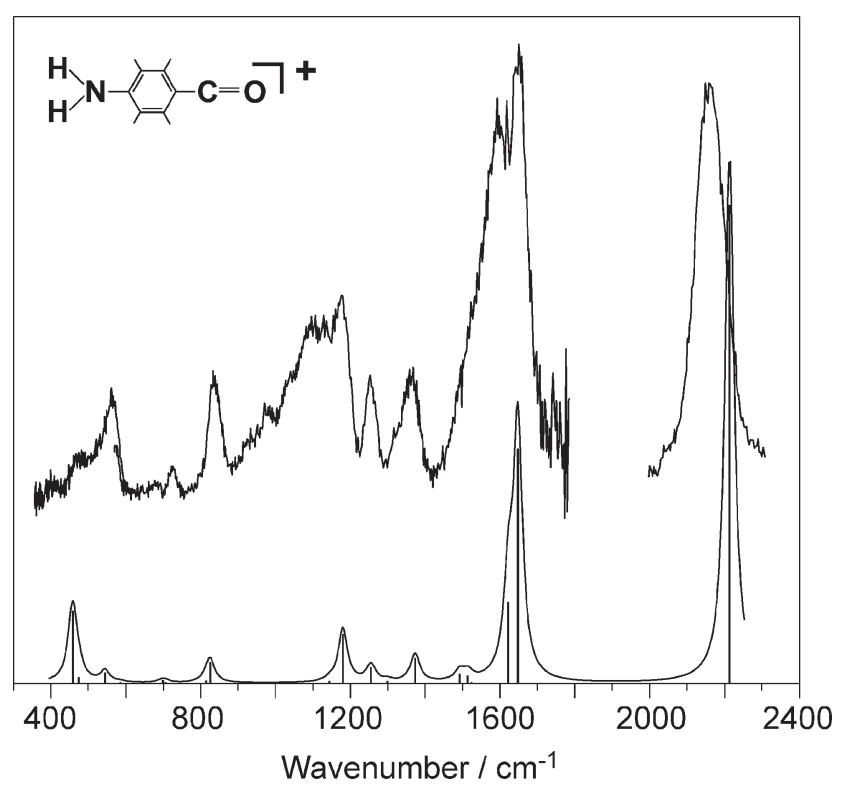

Fig. 3 Infrared spectrum of the PABz cation recorded via FELIX induced IRMPD in an ion trap (thick), compared with a DFT calculation using the B3LYP functional and the D95(d,p) basis set (thin).
The $1500-1700 \mathrm{~cm}^{-1}$ region. This spectral region is dominated by two strong bands at 1655 and $1681 \mathrm{~cm}^{-1}$, which are barely resolved in the calculated spectrum due to the 35 $\mathrm{cm}^{-1}$ Lorentzian convolution. In the experimental spectrum, however, the spacing between the bands is substantially larger $\left(\approx 55 \mathrm{~cm}^{-1}\right.$ ), which may well be due to anharmonic repulsion. As seen in Table 2, both modes have $\mathrm{a}_{1}$ vibrational symmetry and both have strong $\mathrm{NH}_{2}$ scissor character, i.e. both modes are well localized, which makes them susceptible to strong anharmonic interaction. In Fig. 4, it is shown that an interaction matrix element of about $29 \mathrm{~cm}^{-1}$ induces a repulsion between the two bands increasing their spacing to what is observed experimentally. The infrared spectra of partly deuterated $\mathrm{PABz}^{+}$(see below), in which the $\mathrm{NH}_{2}$ scissoring modes are shifted, support the suggestion of anharmonic repulsion.

The 2000-2300 $\mathrm{cm}^{-1}$ region. This range was recorded with FELIX lasing at the third harmonic and different experimental settings. Therefore, the observed relative intensity of the $\mathrm{CO}$ stretching mode with respect to intensities in the rest of the spectrum, is difficult to determine exactly, which likely causes the intensity deviation between experiment and theory. As a consequence of the removal of the unpaired electron on the carbonyl $\mathrm{C}$ atom $\S$ upon ionization, the $\mathrm{CCO}$ angle becomes $180^{\circ}$ and the $\mathrm{C}=\mathrm{O}$ bond becomes substantially stiffer. This causes the $\mathrm{CO}$ stretching band to be blue shifted with respect to the typical $\mathrm{CO}$ stretching band in $\mathrm{sp}^{2}$ hybridized systems, where the band is typically located around $1800 \mathrm{~cm}^{-1}$. In addition, from the calculations one notices a dramatically enhanced absolute intensity compared with the PABA cation.

\subsection{Infrared spectra of deuterated $\mathbf{P A B z}{ }^{+}$}

In an attempt to clarify some ambiguities in the $\mathrm{PABz}^{+}$spectrum, mainly around 1100 and $1600 \mathrm{~cm}^{-1}$, the infrared spectra of partly deuterated $\mathrm{PABz}^{+}$species were recorded between 750 and $1750 \mathrm{~cm}^{-1}$. PABz with a partially D-substituted amino

$\S$ The DFT calculation of the neutral PABz radical shows that the largest spin density resides on the carbonyl $\mathrm{C}$ atom (0.66). 
Table 2 Infrared spectrum of the para-amino benzoyl cation

\begin{tabular}{|c|c|c|c|c|c|c|}
\hline \multirow[b]{2}{*}{ Mode $^{b}$} & \multirow[b]{2}{*}{ Symmetry } & \multicolumn{2}{|c|}{$\mathrm{B} 3 \mathrm{LYP} / \mathrm{D} 95(\mathrm{~d}, \mathrm{p})^{a}\left(C_{2 \mathrm{v}}\right)$} & \multicolumn{2}{|c|}{ Observed } & \multirow{2}{*}{$\begin{array}{l}\mathrm{O}-\mathrm{C} \\
\%\end{array}$} \\
\hline & & $\nu$ & $\operatorname{Int}^{c}$ & $\nu$ & $\operatorname{Int}^{c}$ & \\
\hline$\beta^{\prime}\left(\mathrm{NH}_{2}\right)$ & $b_{1}$ & 459 & 211 & 476 & 0.11 & 3.7 \\
\hline$\beta^{\prime}(\mathrm{NH}) \beta^{\prime}(\mathrm{CH})$ & $b_{1}$ & 474 & 18 & & & \\
\hline$\beta^{\prime}(\mathrm{CH}) \beta^{\prime}(\mathrm{CCO})$ & $b_{1}$ & 544 & 30 & 564 & 0.23 & 3.7 \\
\hline$\delta$ (ring) & $a_{1}$ & 700 & 9 & 725 & 0.07 & 3.6 \\
\hline$\delta$ (ring) & $a_{1}$ & 815 & 8 & & & \\
\hline \multirow[t]{5}{*}{$\beta^{\prime}(\mathrm{CH})$} & $b_{1}$ & 825 & 62 & 838 & 0.29 & 1.6 \\
\hline & & & & $\approx 932$ & 0.10 & \\
\hline & & & & $\approx 980$ & 0.18 & \\
\hline & & & & $\approx 1043$ & 0.25 & \\
\hline & & & & $\approx 1100$ & 0.38 & \\
\hline$\beta(\mathrm{CH})$ & $a_{1}$ & 1180 & 144 & 1176 & 0.45 & -0.3 \\
\hline$\beta(\mathrm{CH}) \sigma(\mathrm{CC})$ & $a_{1}$ & 1254 & 46 & 1255 & 0.26 & 0.1 \\
\hline$\beta(\mathrm{CH})$ & $b_{2}$ & 1299 & 6 & 1324 & 0.10 & 1.9 \\
\hline$\beta(\mathrm{CH}) \sigma(\mathrm{CN})$ & $a_{1}$ & 1373 & 74 & 1364 & 0.28 & -0.7 \\
\hline$\sigma(\mathrm{CC}) \beta(\mathrm{CH})$ & $b_{2}$ & 1492 & 26 & $\approx 1505$ & 0.20 & $\approx 0.9$ \\
\hline$\sigma(\mathrm{CN}) \beta(\mathrm{CH})$ & $a_{1}$ & 1513 & 22 & & & \\
\hline$\alpha\left(\mathrm{NH}_{2}\right) \sigma(\mathrm{CC})$ & $a_{1}$ & 1622 & 238 & 1599 & 0.85 & -1.4 \\
\hline$\alpha\left(\mathrm{NH}_{2}\right)$ & $a_{1}$ & 1647 & 388 & 1654 & 1.00 & 0.4 \\
\hline$\sigma(\mathrm{CO})$ & $a_{1}$ & 2214 & 1403 & 2162 & $?^{d}$ & -2.3 \\
\hline
\end{tabular}

${ }^{a}$ DFT frequencies scaled by $0.98 .{ }^{b} \alpha=$ scissor; $\beta^{\prime}=$ out-of-plane bend; $\beta=$ in-plane bend; $\delta=$ deformation; $\sigma=$ stretch. ${ }^{c}$ Calculated intensity in $\mathrm{km} \mathrm{mol}^{-1}$. Observed intensity: relative to $\alpha\left(\mathrm{NH}_{2}\right)$ at $1654 \mathrm{~cm}^{-1}$. ${ }^{d}$ See text.

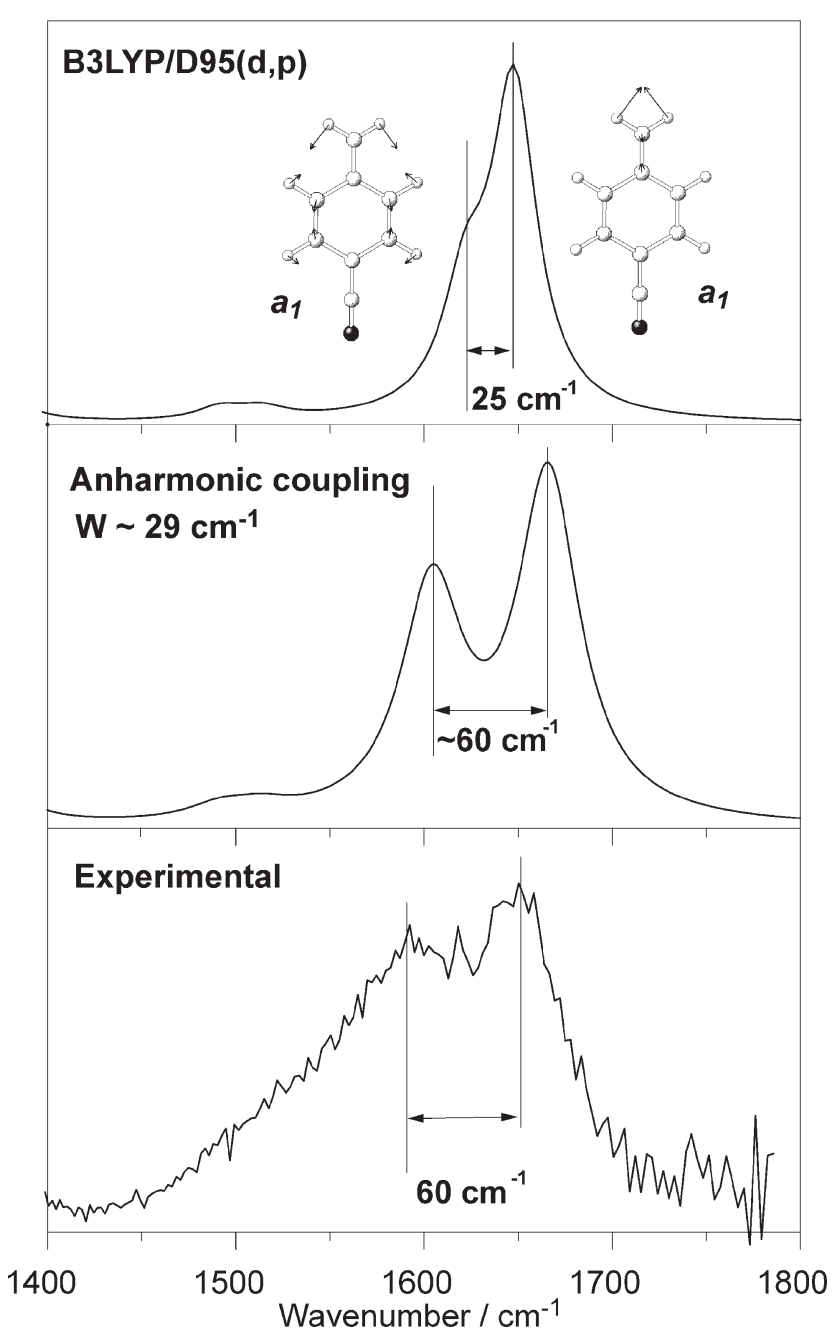

Fig. 4 Zoom of the $\mathrm{PABz}^{+}$spectrum around $1600 \mathrm{~cm}^{-1}$. Introducing interaction between the two $\mathrm{NH}_{2}$ scissoring modes of $\mathrm{a}_{1}$ symmetry yields better agreement with the experiment in terms of the spacing of the two main bands in this region. moiety is obtained by using partially deuterated PABA as precursor. A mass spectrum of the sample reveals the presence of masses 120, 121 and 122 as parent ions in an approximately 3:7:10 ratio. The resolving power of the ion trap is insufficient to isolate one of these masses and therefore the infrared spectra of the three parent ions are recorded simultaneously. Main infrared induced fragments are found at mass channels 94, 93, 92 and $67,66,65$, which can be individually observed as the resolving power of the TOF mass spectrometer is sufficient. From the infrared fingerprints on the different mass channels (not shown), one can conclude that the m94 and m67 fragments have a common parent (presumably m122), m93 and m66 have a common parent (presumably m121) and m92 and $\mathrm{m} 65$ have a common parent, being the fully hydrogenated $\mathrm{PABz}^{+}$at $\mathrm{m} 120$. The spectra shown in Fig. 5 are generated by summing the intensity in the two main fragment channels for each of the three species and band centers for the deuterated species are listed in Table 3. Judging from the unique features in each of the spectra, there appears to be little cross-talk between the channels. This indicates that in the fragmentation process, the $\mathrm{H} / \mathrm{D}$ atoms of the amino-group are likely transfered to the charged fragment, providing a clue to the structure of the m65 fragment (see Section 3.4.).

In the short wavelength region around $1600 \mathrm{~cm}^{-1}$, an interesting difference between the hydrogenated $\mathrm{PABz}^{+}$and the deuterated species is observed. The two bands with strong $\mathrm{NH}_{2}$ scissoring character are closely spaced in $\mathrm{H}_{2}-\mathrm{PABz}^{+}$giving rise to anharmonic repulsion as discussed in the previous section. In the deuterated species, however, the scissoring modes are shifted to longer wavelengths due to the larger reduced mass of the atoms involved in the vibration. Moreover, particularly in the singly deuterated species, the normal modes are not as localized as in the more symmetric species. In the spectral range displayed, the strongest band in the spectra of the deuterated species is now mainly due to CC stretching. The next strongest band around $1570 \mathrm{~cm}^{-1}$ possesses some amino scissoring character. Although the two modes are of the same symmetry, no evidence for anharmonic interaction is observed due to the larger energy spacing between the modes and the more delocalized nature of the vibrations. In contrast to what is observed for the two deuterated species, there does 


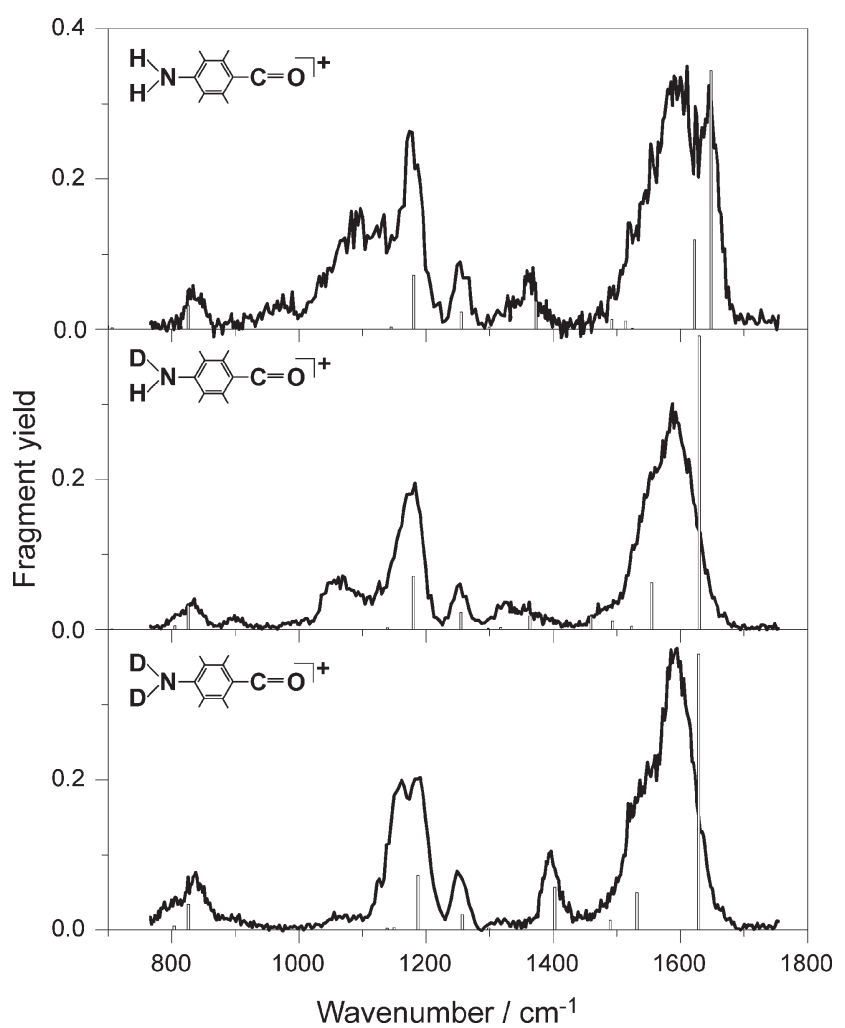

Fig. 5 Simultaneously recorded infrared spectra of singly and doubly deuterated $\mathrm{PABz}^{+}$as well as fully hydrogenated $\mathrm{PABz}^{+}$. For $\mathrm{H}_{2}-\mathrm{PABz}$ fragment channels $\mathrm{m} 92$ and $\mathrm{m} 65$ are summed, for HD-PABz $\mathrm{m} 93$ and $\mathrm{m} 66$, and for $\mathrm{D}_{2}-\mathrm{PABz} \mathrm{m} 94$ and $\mathrm{m} 67$. Stick spectra give B3LYP/ D $95(d, p)$ calculations of the parent species (frequencies scaled by 0.98 ).

not appear to be any red-shift for the $1650 \mathrm{~cm}^{-1}$ band in the hydrogenated species, which is caused by the anharmonic repulsion; the general redshift due to multiple photon excitation is compensated for by a blueshift due to the anharmonic repulsion.

In the $1000-1200 \mathrm{~cm}^{-1}$ range, substantial differences between the three species are observed. As noticed in the previous section, the hydrogenated $\mathrm{PABz}^{+}$spectrum shows several congested bands in this range, which cannot be explained by the DFT calculation. The spectrum of the singly deuterated $\mathrm{PABz}^{+}$shows less congestion in this range and basically shows one band around $1050 \mathrm{~cm}^{-1}$ that is completely absent in the DFT calculation. Note that around $900 \mathrm{~cm}^{-1}$ another weak feature is observed that is unaccounted for by the calculation. The spectrum of the doubly deuterated species is even cleaner in this range although it appears that the weak bands calculated around $1150 \mathrm{~cm}^{-1}$ are enhanced in the experimental spectrum, which may well be due to the presence of the strong in-plane $\mathrm{CH}$ bending band at $1186 \mathrm{~cm}^{-1}$. Such behavior in IRMPD spectra, where weak bands in the red tail of a strong band are enhanced in intensity, has been observed and explained previously. ${ }^{22,30}$ These striking spectral differences in the spectra of the different deuterated species, lead to the conclusion that the extra structure around $1100 \mathrm{~cm}^{-1}$ in the $\mathrm{H}_{2}-\mathrm{PABz}^{+}$spectrum is due to the amino unit.

The observations in partly deuterated $\mathrm{PABz}$ clearly suggest that for a better understanding of the hydrogenated $\mathrm{PABz}^{+}$ spectrum, the vibrational modes involving the amino group require special attention. Although virtually nothing is known about this mode in $\mathrm{PABz}$, the situation appears to be rather similar to that in aniline, which has been studied in great detail in both the neutral and cationic state. ${ }^{25,34-39}$ Neutral ground state aniline is bent and the $\mathrm{NH}_{2}$ inversion mode shows a symmetric double-well potential. In the $\mathrm{S}_{1}$ electronically excited state, the molecule is planar and the mode is known to be a large amplitude strongly anharmonic vibration. The situation for the cation is similar to that for the $S_{1}$ state of the neutral. In general, the strongly anharmonic vibrational potentials in these systems cause a frequency shift and an intensity drop of the fundamental transition as well as a strongly enhanced intensity of the overtone transition.

For $\mathrm{PABz}^{+}$, we used GAUSSIAN 98 to perform a potential scan along the $\mathrm{NH}_{2}$ inversion coordinate, which yields a curve that can be fitted to the sum of a quadratic function $A q^{2}$ and a quartic function $B q^{4}$ with $A \approx B$ (see Fig. 6). If one determines the eigenvalues in this fitted potential and scales the fundamental transition to the observed value of $476 \mathrm{~cm}^{-1}$, the first overtone is found at $1059 \mathrm{~cm}^{-1}$. This is well in the range where the strongly congested additional structure is observed. Although analysis of the fitted potential predicts a negligible intensity for the overtone, it was found for cationic aniline that the observed intensity of the overtone transition is much stronger than what is expected from such a simple empirical potential. ${ }^{25,40}$ It was suggested that the inversion mode is particularly susceptible to anharmonic interaction with nearby modes, which could enhance its activity. The situation in the $\mathrm{PABz}$ cation appears to be rather similar.

Table 3 Infrared spectra of partly deuterated $\mathrm{PABz}^{+}$

\begin{tabular}{|c|c|c|c|c|c|c|c|c|c|}
\hline \multicolumn{5}{|c|}{$\mathrm{D}_{2}-\mathrm{PABz}$} & \multicolumn{5}{|c|}{$\mathrm{D}_{1}-\mathrm{PABz}$} \\
\hline \multicolumn{3}{|c|}{ B3LYP/D95(d,p) } & \multicolumn{2}{|c|}{ Observed } & \multicolumn{3}{|c|}{ B3LYP/D95(d,p) } & \multicolumn{2}{|c|}{ Observed } \\
\hline$C_{2 \mathrm{v}}$ & $\nu^{a}$ & $\operatorname{Int}^{b}$ & $\nu$ & Int & $C_{\mathrm{s}}$ & $\nu^{a}$ & $\operatorname{Int}^{b}$ & $\nu$ & $\operatorname{Int}^{b}$ \\
\hline$a_{1}$ & 804 & 11 & 797 & 0.08 & $a^{\prime}$ & 805 & 10 & 807 & 0.03 \\
\hline \multirow[t]{3}{*}{$\mathrm{b}_{1}$} & 825 & 69 & 836 & 0.20 & $\mathrm{a}^{\prime \prime}$ & 825 & 65 & 831 & 0.12 \\
\hline & & & 896 & 0.03 & & & & 899 & 0.05 \\
\hline & & & 1070 & 0.03 & & & & 1058 & 0.25 \\
\hline$a_{1}$ & 1149 & 7 & 1158 & 0.43 & & & & 1090 & 0.10 \\
\hline$a_{1}$ & 1187 & 145 & 1186 & 0.46 & $\mathrm{a}^{\prime}$ & 1180 & 142 & 1176 & 0.72 \\
\hline$a_{1}$ & 1256 & 41 & 1251 & 0.19 & $\mathrm{a}^{\prime}$ & 1254 & 46 & 1252 & 0.19 \\
\hline$a_{1}$ & 1402 & 113 & 1395 & 0.27 & $\mathrm{a}^{\prime}$ & 1317 & 6 & 1322 & 0.10 \\
\hline $\mathrm{b}_{2}$ & 1489 & 26 & & & $\mathrm{a}^{\prime}$ & 1363 & 35 & 1358 & 0.08 \\
\hline \multirow[t]{2}{*}{$\mathrm{a}_{1}$} & 1531 & 99 & $\approx 1525$ & 0.33 & $\mathrm{a}^{\prime}$ & 1459 & 35 & & \\
\hline & & & $\approx 1552$ & 0.40 & $\mathrm{a}^{\prime}$ & 1493 & 22 & 1482 & 0.07 \\
\hline \multirow[t]{2}{*}{$a_{1}$} & 1629 & 735 & 1592 & 1.00 & $\mathrm{a}^{\prime}$ & 1554 & 125 & 1559 & 0.62 \\
\hline & & & & & $\mathrm{a}^{\prime}$ & 1630 & 782 & 1590 & 1.00 \\
\hline
\end{tabular}

${ }^{a}$ DFT frequencies scaled by $0.98 .{ }^{b}$ Only bands with $I>5 \mathrm{~km} / \mathrm{mol}$ are listed. Observed intensity: relative 


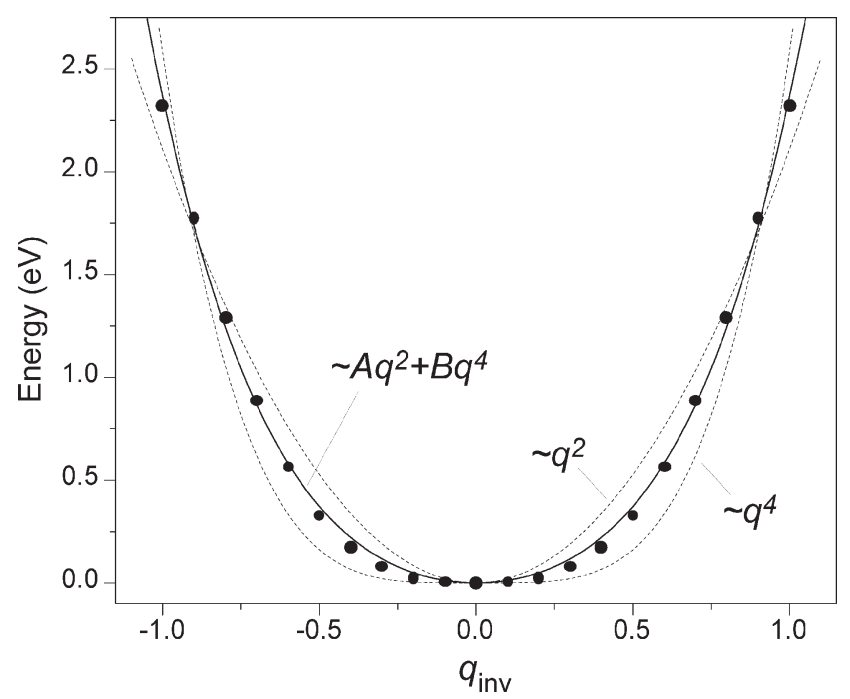

Fig. 6 Potential scan of the $\mathrm{NH}_{2}$ out-of-plane bending mode in $\mathrm{PABz}^{+}$, revealing a strongly anharmonic behavior. The potential is best approximated by a combined quadratic/quartic function, where the coefficients are similar $(A / B \approx 1.02)$.

\subsection{Infrared dissociation dynamics of the PABz cation}

Upon infrared multiple photon excitation, the PABA cation (m137) fragments into channels $\mathrm{m} 120$ and m92. The PABz cation (m120) shows fragmentation into channels $\mathrm{m} 92$ and m65. In Fig. 7, the different dissociation pathways are outlined. Values for the various dissociation energies are estimated from DFT calculations using the B3LYP functional and the D95(d,p) basis set. ${ }^{32}$ Zero point energy corrected structural energies are calculated at their optimized geometries so that possible barriers in the reaction channels are neglected. Note that for some of the values in the scheme experimental values have been reported, ${ }^{31}$ however, for the sake of consistency, we use only calculated values here.

The main dissociation product $(>80 \%)$ of $\mathrm{PABA}^{+}$is $\mathrm{PABz}^{+}$, which is formed by dehydroxylation. The minor $\mathrm{m} 92$ fragmentation product is formed by detachment of the acidic side group, leaving behind an aniline-like cation $\mathrm{C}_{6} \mathrm{H}_{4} \mathrm{NH}_{2}{ }^{+}$. This fragment may rearrange to the energetically more favorable $\mathrm{C}_{6} \mathrm{H}_{5} \mathrm{NH}^{+}$form $(\Delta E \approx-1.0 \mathrm{eV})$.

For the $\mathrm{PABz}^{+}$parent ion, the mass 92 fragment is again the aniline-like cation $\mathrm{C}_{6} \mathrm{H}_{4} \mathrm{NH}_{2}{ }^{+}$, which can be formed by
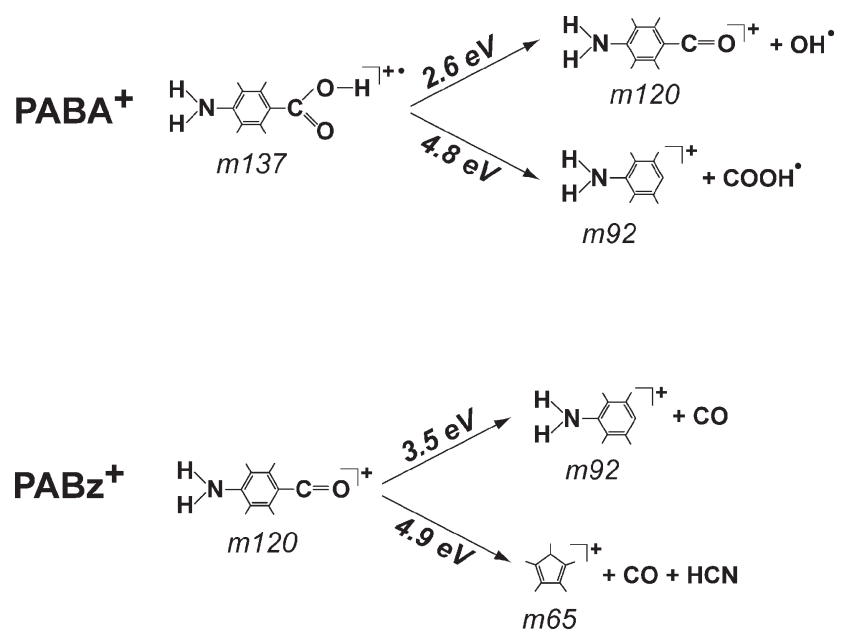

Fig. 7 Infrared photodegradation pathways of the PABA and PABz cations observed in the experiments. Dissociation energies are estimated from DFT calculations at the optimized structures indicated. The structure of the m65 fragment is tentative. abstraction of the $\mathrm{CO}$ unit. For the mass 65 fragment several possible structures are conceivable, mainly the quasilinear $\mathrm{HC}_{4} \mathrm{NH}_{2}^{+}$and $\mathrm{NC}_{4} \mathrm{H}_{3}^{+}$species, the cyanoallene $\left(\mathrm{NCCHCCH}_{2}{ }^{+}\right)$and the cyclopentadienyl ion $\left(\mathrm{C}_{5} \mathrm{H}_{5}{ }^{+}\right)$. From the experiments on $\mathrm{PABz}^{+}$with a deuterated amino-group, we conclude that the deuterium atoms are conserved in the charged fragment, which indicates that either the $\mathrm{NH}_{2}$ unit is conserved in the charged fragment or that the H-atoms are detached from the nitrogen atom, which forms a cyano-group $(\mathrm{C} \equiv \mathrm{N})$ in the uncharged fragment(s). We assume this latter scenario to be the actual case and the charged fragment to be the $\mathrm{C}_{5} \mathrm{H}_{5}{ }^{+}$ion $\uparrow$ since it yields (a) the lowest energy dissociation channel, (b) only closed-shell fragments and (c) the charge on the fragment with the lowest IP. Note that instead of the neutral $\mathrm{CO}$ and $\mathrm{HCN}$ products, formyl cyanide ( $\mathrm{HCOCN}$ ) may be formed, however, this molecule is known to be unstable $^{41}$ and the calculated dissociation energy is the same within $0.1 \mathrm{eV}$. In any case, for the following qualitative discussion, the exact structure of the m65 fragment is immaterial as long as we assume the threshold for dissociation to be higher than that for the $\mathrm{m} 92$ fragment channel. Starting from $\mathrm{PABz}^{+}$, it is clear that the m92 fragment can be formed in a unimolecular dissociation process. However, the situation is less clear for the formation of the m65 dissociation product. It may be formed in a unimolecular fashion as well, however, it is also conceivable that two consecutive dissociation reactions occur. In the latter case, additional photon absorption could take place after the first dissociation reaction in order to overcome the next dissociation threshold. However, in the following we present experimental evidence, which excludes photon absorption by the reaction intermediate. Moreover, it is experimentally shown that the dissociation qualitatively follows statistical unimolecular dissociation dynamics.

In Fig. 8 the infrared spectrum of the $\mathrm{CO}$ stretching fundamental, representing an isolated spectral feature in the $\mathrm{PABz}^{+}$ spectrum (see Fig. 3), is shown as recorded on the two fragmentation channels 92 and 65 . Two things can be readily observed: (1) the spectrum in the m65 channel is clearly redshifted with respect to that in the m92 channel, and (2) the relative intensities in the two channels change upon changing the laser pulse energy. It is important to note that, different from the PABz cation, the mass 92 fragment does not possess a $\mathrm{CO}$ chromophore and therefore possesses no absorption bands in the wavelength range plotted here. This excludes a sequential absorption process, where after formation the mass 92 fragment absorbs additional photons to overcome the second dissociation threshold leading to the mass 65 fragment. The spectral shift between the two fragment channels is caused by cross-anharmonicities of the $\mathrm{CO}$ stretching mode with all other modes, which shifts the absorption frequency to longer wavelengths as the internal energy of the ion increases. Monitoring the m65 fragment channel, one observes the PABz cation at a higher average internal energy than when monitoring the m92 channel.

Recording the spectrum on the $\mathrm{CO}$ stretch fundamental for different laser pulse energies reveals a textbook example of a statistical dissociation process, which can be described by simple transition state theory. ${ }^{5,42,43}$ As a result of infrared multiple photon absorption, the internal energy of the PABz cation increases and as it exceeds the dissociation threshold for the formation of mass 92, a competition between the unimolecular dissociation rate and the photon absorption rate occurs. The photon absorption rate is determined by the infrared laser fluence and the dissociation rates are determined by the internal energy. Further photon absorption leads to even higher internal energies eventually exceeding the second dissociation threshold leading to the mass 65 fragment. At low internal

I Or the $\mathrm{C}_{5} \mathrm{H}_{4} \mathrm{D}^{+}$or $\mathrm{C}_{5} \mathrm{H}_{3} \mathrm{D}_{2}{ }^{+}$ions for the singly and doubly deuterated species, respectively. 


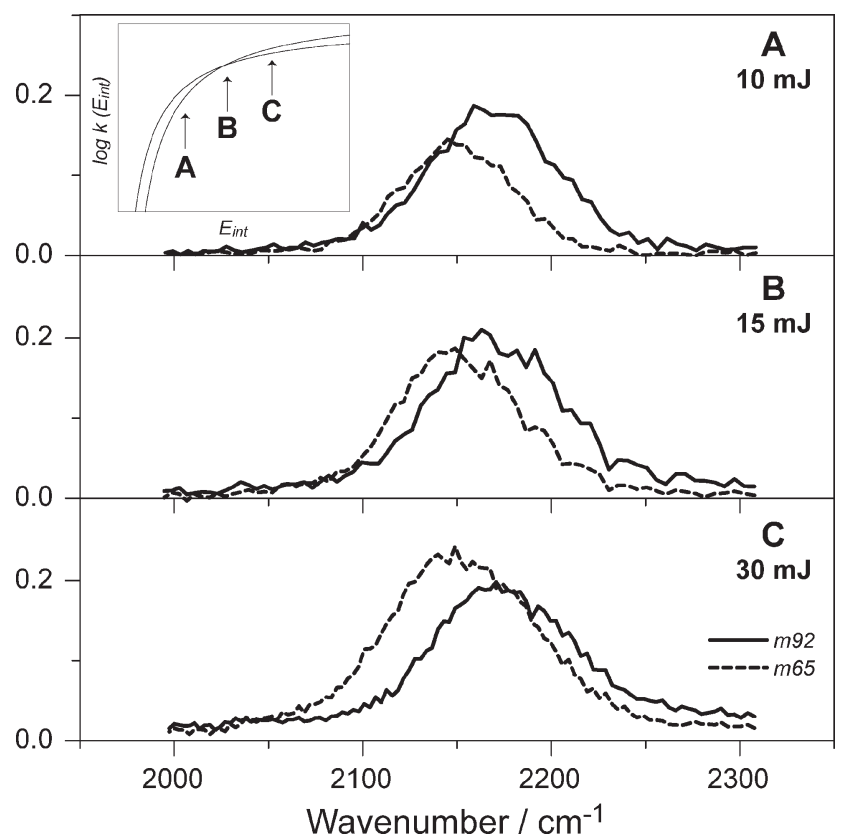

Fig. 8 Infrared spectra of the PABz cation recorded on the 92 (solid) and 65 (dashed) fragment mass channels in the CO stretching region for three different FELIX pulse energies. Dissociation energies for the 92 and 65 fragment channels are calculated to be 3.5 and $4.9 \mathrm{eV}$, respectively. Hence, the higher energy required to reach the 65 channel is evidenced by the larger redshift, induced by anharmonicity. The inset shows a generic picture of a statistical dissociation rate constant $k$ as function of internal energy $E_{\text {int }}$ for two different dissociation channels. The arrows qualitatively indicate the positions where the three different spectral scans are located.

energies, the lowest energy exit channel possesses the highest decay rate. However, increasing the internal energy, the higher exit channel becomes available and its rate eventually exceeds that of the lower energy exit channel (see the inset in Fig. 8). This behaviour is commonly described by a pre-exponential factor in the expression for the unimolecular dissociation rate, which is on the order of the vibrational frequency of the dissociation coordinate. ${ }^{5,42}$ Hence, in this simple picture, the lowest energy dissociation coordinate is associated with the floppiest, i.e. lowest energy mode and higher energy dissociation channels have a higher frequency factor, causing the cross-over of the rate constants at higher internal energies.

For a good understanding of the observations, the $\mathrm{CO}$ stretching band is particularly well-suited because of its isolated location in the spectrum. In a more dense spectrum, the situation becomes more complicated. For instance, in addition to the effects of multiple photon absorption discussed above, closely spaced bands may give rise to enhanced IRMPD intensity as was shown in previous experiments ${ }^{22}$ as well as model calculations. ${ }^{30}$ During the multiple photon excitation process, the molecular resonance usually shifts to longer wavelengths as a consequence of the increasing internal energy. Eventually, resonance with the narrow-band laser radiation will be lost unless a second band, located slightly to the blue, simultaneously shifts into resonance, thus enhancing the multiple photon efficiency. In Fig. 9, the spectra of the PABz cation as observed on the individual fragment channels $\mathrm{m} 92$ and $\mathrm{m} 65$ are shown. Significant differences induced by the complex multiple photon excitation process are noticed, particularly on strong and congested bands. This example indicates that in IRMPD spectroscopy it is of importance to record all fragment mass channels. Note also that contrary to the $\mathrm{CO}$ stretching region (Fig. 8), a sequential absorption process, which could also give rise to differences in the spectra recorded on the two fragment channels, can strictly not be excluded here.

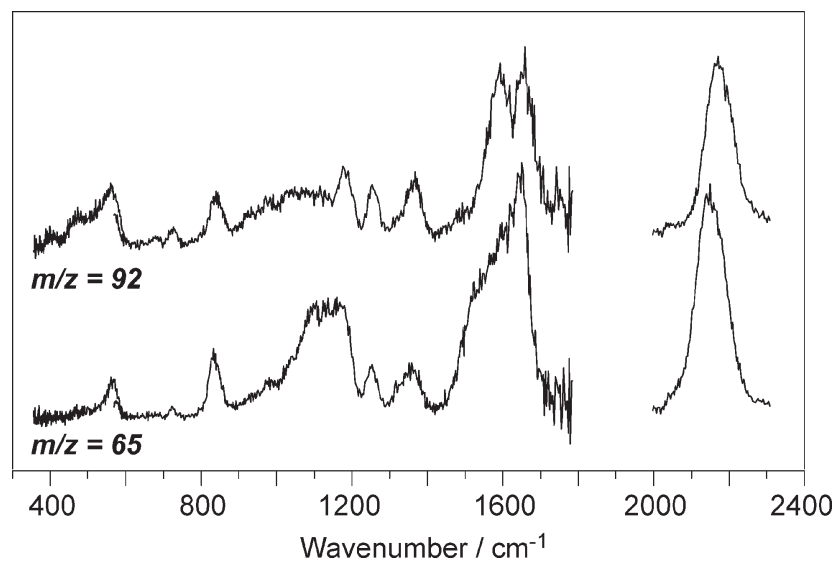

Fig. 9 Infrared spectra of the PABz cation as recorded on the individual fragment channels $\mathrm{m} 92$ and $\mathrm{m} 65$ (see Fig. 6). Note the differences in the spectra particularly in the vicinity of strong and/or congested bands.

In a recent publication, ${ }^{30}$ we have shown that using IRMPD model calculations, ${ }^{4,44}$ one can retrieve the mode-dependent anharmonicities by comparing the IRMPD and linear absorption spectra. It is anticipated that if two different dissociation pathways exist, these parameters can be determined with even much better accuracy. At this moment, however, such an analysis is rather speculative for the PABz cation since neither linear absorption data nor accurate dissociation parameters, such as the rate of dissociation as a function of internal energy, are known.

\section{Conclusions and outlook}

The infrared spectra of the PABA cation as well as its dehydroxylated closed-shell fragment ion, $\mathrm{PABz}^{+}$, have been recorded for the first time. Comparison with DFT calculations shows that the spectra, particularly that of $\mathrm{PABz}^{+}$, deviate from theory. Deuteration of the amino-group indicates that the discrepancies are related to the strongly anharmonic inversion mode (i.e. the $\mathrm{NH}_{2}$ out-of-plane bending mode). Both in the PABA as well as in the PABz cation, the situation appears to be rather similar to that in cationic aniline. Note also that the the geometry of the $\mathrm{PABz}$ cation represents a hybrid between the previously studied aniline cation, which is planar, and benzoyl cation, which possesses a $180^{\circ} \mathrm{CCO}$ bond.

Dissociation dynamics of the PABz cation, for which two decay channels with different exit barriers are available, are in good qualitative agreement with what is predicted by statistical theory. Moreover, the effects of vibrational anharmonicity in this process are spectrally resolved here. It also indicates that the appearance of an IRMPD spectrum of one species may show significant differences depending on which fragment channel is monitored. However, these effects are small and frequency shifts are on the order of $1-2 \%$ in this example.

For the study of ionic fragments, mass selective isolation prior to infrared dissociation is an important issue. In this study, we used the mass selective instability properties of the quadrupole trap (in particular the low-mass-cut-off at $a_{z}=0$ and $q_{z}=0.908^{10,11}$ ), which allows only to isolate the heaviest species. Since the ArF laser induces complete fragmentation of PABA, the PABz fragment cation is the heaviest ionic species present in the trap. However, for a more general study of ionic fragments one would like to be able to isolate any fragment ion, i.e. one would like to eject ions with both higher and lower mass than the target ion. This can be accomplished via apex isolation ${ }^{10,45}$ or resonant excitation of the 
mass-dependent secular ion frequency, ${ }^{10,11}$ which we intend to implement in our apparatus in the near future.

\section{Acknowledgements}

We thank Dr B.G. Sartakov for the various discussions on multiple photon excitation. This work is part of the research program of FOM, which is financially supported by the Nederlandse Organisatie voor Wetenschappelijk Onderzoek (NWO).

\section{References}

1 M. A. Duncan, Int. J. Mass Spectrom., 2000, 200, 545-569; and references therein.

2 S. P. Ekern, A. G. Marshall, J. Szczepanski and M. Vala, J. Phys. Chem. A, 1998, 102, 3498.

3 J. G. Black, E. Yablonovitch, N. Bloembergen and S. Mukamel, Phys. Rev. Lett., 1977, 38, 1131-1134.

4 E. R. Grant, P. A. Schulz, Aa. S. Sudbo, Y. R. Shen and Y. T. Lee, Phys. Rev Lett., 40, 115-118.

5 V. N. Bagratashvili, V. S. Letokhov, A. A. Makarov and E. A. Ryabov, Multiple Photon Infrared Laser Photophysics, Photochemistry, Harwood Academic Publishers, Chur, Switzerland, 1985.

6 S. S. Alimpiev and B. G. Sartakov, Laser Chem., 1992, 12, 147-172.

7 R. D. F. Settle and T. R. Rizzo, J. Chem. Phys., 1992, 92, 2823-2825

8 J. L. Lyman, B. E. Newman, J. W. Early and A. F. G. van der Meer, J. Phys. Chem. A, 1997, 101, 49-54

9 W. Paul, Rev. Mod. Phys., 1990, 62, 531-540.

10 R. E March and R. J. Hughes, Quadrupole Storage Mass Spectrometry, John Wiley \& Sons, Inc., New York, 1989.

11 R. E. March, J. Mass Spectrom., 1997, 32, 351-369.

12 S. K. Shin and J. L. Beauchamp, J. Am. Chem. Soc., 1990, 112, 2066-2069.

13 D. M. Peiris, M. A. Cheeseman, R. Ramanathan and J. R. Eyler, J. Phys. Chem., 1993, 97, 7839-7843.

14 R. C. Dunbar, Int. J. Mass Spectrom., 2000, 200, 571-589.

15 T. Sawamura, A. Fujii, S. Sato, T. Ebata and N. Mikami, J. Phys. Chem., 1996, 100, 8131-8138.

16 N. Solcà and O. Dopfer, J. Am. Chem. Soc., 2003, 125, 1421-1430.

17 J. Oomens, A. J. A. van Roij, G. Meijer and G. von Helden, Astrophys. J., 2000, 542, 404-410.

18 K. R. Asmis, M. Brümmer, C. Kaposta, G. Santambrogio, G. von Helden, G. Meijer, K. Rademann and L. Wöste, Phys. Chem. Chem. Phys., 2002, 4, 1101-1104.

19 K. R. Asmis, N. L. Pivonka, G. Santambrogio, M. Brümmer, C. Kaposta, D. M. Neumark and L. Wöste, Science, 2003, 299 1375-1377.

20 J. Lemaire, P. Boissel, M. Heninger, G. Mauclaire, G. Bellec, H. Mestdagh, A. Simon, S. Le Caer, J. M. Ortega, F. Glotin and P. Maitre, Phys. Rev. Lett., 2002, 89, 273002.

21 D. T. Moore, J. Oomens, A. F. G. van der Meer, G. von Helden, G. Meijer, J. Valle, A. G. Marshall and J. R. Eyler, to be published.
22 J. Oomens, J. M. Bakker, B. G. Sartakov, G. Meijer and G. von Helden, Chem. Phys. Lett., 2003, 367, 576-580.

23 F. Wöhler and J. Liebig, Ann. Pharm. (Lemgo, Ger.), 1832, 3, 249-282

24 G. Meijer, M. S. de Vries, H. E. Hunziker and H. R. Wendt, J. Chem. Phys., 1990, 92, 7625-7635.

25 H. Piest, G. von Helden and G. Meijer, J. Chem. Phys., 1999, 110, 2010-2015.

26 J. Oomens, et al., to be published.

27 M. Putter, G. von Helden and G. Meijer, Chem. Phys. Lett., 1996 , 258, 118-122.

28 S. M. Michael, M. Chien and D. M. Lubman, Rev. Sci. Instrum., 1992, 63, 4277-4284.

29 D. Oepts, A.F.G. van der Meer and P. W. van Amersfoort, Infrared Phys. Technol., 1995, 36, 297-308. See also http://www.rijnh.nl/felix.

30 J. Oomens, A. G. G.M. Tielens, B. G. Sartakov, G. von Helden and G. Meijer, Astrophys. J., 2003, 591, 968-985.

31 F. Benoit, Org. Mass Spectrom., 1973, 7, 295-303.

32 M. J. Frisch, G. W. Trucks, H. B. Schlegel, G. E. Scuseria, M. A Robb, J. R. Cheeseman, V. G. Zakrzewski, J. A. Montgomery, Jr., R. E. Stratmann, J. C. Burant, S. Dapprich, J. M. Millam, A. D. Daniels, K. N. Kudin, M. C. Strain, O. Farkas, J. Tomasi, V. Barone, M. Cossi, R. Cammi, B. Mennucci, C. Pomelli, C. Adamo, S. Clifford, J. Ochterski, G. A. Petersson, P. Y. Ayala Q. Cui, K. Morokuma, D. K. Malick, A. D. Rabuck, K Raghavachari, J. B. Foresman, J. Cioslowski, J. V. Ortiz, B. B. Stefanov, G. Liu, A. Liashenko, P. Piskorz, I. Komaromi, R. Gomperts, R. L. Martin, D. J. Fox, T. Keith, M. A. Al-Laham, C. Y. Peng, A. Nanayakkara, C. Gonzalez, M. Challacombe, P. M. W. Gill, B. G. Johnson, W. Chen, M. W. Wong, J. L. Andres, M. Head-Gordon, E. S. Replogle and J. A. Pople, GAUSSIAN 98 (Revision A.7), Gaussian, Inc., Pittsburgh, PA, 1998.

33 S. R. Langhoff, J. Phys. Chem., 1996, 100, 2819-2841.

34 M. Takahashi, H. Ozeki and K. Kimura, J. Chem. Phys., 1992, 96, 6399-6406.

35 X. Zhang, J. M. Smith and J. L. Knee, J. Chem. Phys., 1992, 97, 2843-2860.

36 X. Song, M. Yang, E. R. Davidson and J. R. Reilly, J. Chem. Phys., 1993, 99, 3224-3233.

37 W. E. Sinclair and D. W. Pratt, J. Chem. Phys., 1996, 105, 7942-7956.

38 P. M. Wojciechowski, W. Zierkiewicz, D. Michalska and P. Hobza, J. Chem. Phys., 2003, 118, 10900-10911.

39 J. M. Hollas, M. R. Howson, T. Ridley and L. Halonen, Chem. Phys. Lett., 1983, 98, 611-614.

40 J. A. Piest, PhD Thesis, University of Nijmegen, 2002.

41 D. J. Clouthier and D. C. Moule, J. Am. Chem. Soc., 1987, 109, 6259-6261.

42 K. A. Holbrook, M. J. Pilling and S. H. Robertson, Unimolecular Reactions, John Wiley \& Sons, Chichester, 1971.

43 R. D. Levine and R. B. Bernstein, Molecular Reaction Dynamics and Chemical Reactivity, Oxford University Press, Oxford, 1985, $432 \mathrm{ff}$.

44 G. von Helden, I. Holleman, G. Meijer and B. Sartakov, Opt. Exp., 1999, 4, 46-52.

45 See e.g. J. F. J. Todd and A. D. Penman, Int. J. Mass Spectrom. Ion Proc., 1991, 106, 1-20. 